\title{
SUSCEPTIBILIDAD DE Tetranychus urticae Koch (Acari: Tetranychidae) COLECTADA EN Primula obconica Hance Y Convolvulus arvensis L. A ACARICIDAS
}

\author{
Susceptibility of Tetranychus urticae Koch (Acari: Tetranychidae) collected in \\ Primula obconica Hance and Convolvulus arvensis $\mathbf{L}$. to acaricides
}

\author{
Alberto Flores F. ${ }^{1}$, Gonzalo Silva A. ${ }^{1 *}$, Maritza Tapia V. ${ }^{1}$, Pedro Casals B. ${ }^{1}$
}

\begin{abstract}
A B S T R A C T
The use of acaricides of high toxicity in the production and handling of ornamental plants should be a matter of consumer concern. The susceptibility of two populations of Tetranychus urticae was assessed: one collected from Primula obconica Hance and the other from Convolvulus arvenis $\mathrm{L}$. as susceptible reference strain to the acaricides dicofol, abamectin alone and mixed with the surfactant phosphatidylcholine, azadirachtin, sulfur, pyridaben and fenazaquin. The evaluated parameters were mortality, lethal concentration $50 \%\left(\mathrm{LC}_{50}\right)$ and $90 \%\left(\mathrm{LC}_{90}\right)$, lethal time $50 \%\left(\mathrm{LT}_{50}\right)$ and $90 \%\left(\mathrm{LT}_{90}\right)$, effectiveness and index of resistance (IR). The experimental design was completely at random. Each treatment had 1/4, 1/2, 1, 2 and $4 \mathrm{X}$ of the doses indicated by the manufacturer, five replicates and were sprayed with a Potter spray tower. The results showed that the $\mathrm{LC}_{50}$ for azadirachtin and sulfur was significantly high. Regarding the population from $P$. obconica, abamectin alone and in mixture with phosphatidylcholine and fenazaquin showed the highest efficiency. Azadirachtin and sulfur were the acaricides showing the slowest activity and the resistance index indicated that the population collected in P. obconica should be considered susceptible to all the evaluated acaricides.
\end{abstract}

Key words: tolerance, twospotted spider mite, mites.

\section{R E S U M E N}

El uso de acaricidas de alta toxicidad en la producción y manejo de plantas ornamentales es un tema que debería preocupar a los consumidores. Se evaluó la susceptibilidad en laboratorio de dos poblaciones de Tetranychus urticae colectadas una en Primula obconica Hance y la otra en Convolvulus arvenis L., que se usó como raza sensible de referencia, a dicofol, abamectina, sola y en mezcla con el surfactante fosfatidilcolina, azadirachtina, azufre, pyridaben y fenazaquin. Las variables evaluadas fueron mortalidad, concentración letal $50 \%\left(\mathrm{CL}_{50}\right)$ y $90 \%\left(\mathrm{CL}_{90}\right)$, tiempo letal $50 \%\left(\mathrm{TL}_{50}\right)$ y $90 \%\left(\mathrm{TL}_{90}\right)$, eficacia e índice de resistencia (IR). El diseño experimental fue completamente al azar. Cada acaricida se evaluó en 1/4, 1/2, 1,2 y 4 veces la dosis recomendada según el fabricante, y cada tratamiento tuvo cinco repeticiones y se aplicaron con una torre de Potter. Los resultados muestran que para azadirachtina y azufre la $\mathrm{CL}_{50}$ fue mayor. En la población proveniente de $P$. obconica, abamectina sola y en mezcla con fosfatidilcolina y fenazaquin propiciaron la mayor eficiencia. Azadirachtina y azufre demostraron ser los acaricidas de más lenta acción y el índice de resistencia indica que la población colectada en $P$. obconica debe ser considerada como sensible a todos los acaricidas evaluados.

Palabras clave: tolerancia, arañita bimaculada, ácaros.

\footnotetext{
${ }^{1}$ Universidad de Concepción, Facultad de Agronomía, Vicente Méndez 595, Casilla 537, Chillán, Chile.

E-mail: gsilva@udec.cl *Autor para correspondencia.

Recibido: 10 de febrero de $2006 . \quad$ Aceptado: 21 de junio de 2006.
} 


\section{INTRODUCCIÓN}

La arañita bimaculada (Tetranychus urticae Koch; Acari: Tetranychidae) es considerada uno de los ácaros plaga de mayor importancia a escala mundial (González, 1989). El daño provocado por este ácaro consiste en la remoción del contenido celular, quedando la célula prácticamente vacía, con escaso contenido de material intracelular, dando un aspecto de hoja con puntuaciones cloróticas y bronceada (University of California, 2002). Sadrás et al. (1998) indican que T. urticae se alimenta principalmente del mesófilo, reduciendo significativamente la resistencia estomática, la fotosíntesis y la tasa respiratoria, afectando negativamente la tasa de absorción energética de la planta.

El daño de este ácaro es especialmente importante en cultivos ornamentales debido a que un mínimo daño en cualquier estructura afecta fuertemente la calidad perdiendo su valor comercial (University of California, 2002).

El control de T. urticae en ornamentales y en la mayoría de los cultivos, se realiza casi exclusivamente con agroquímicos (Takematsu et al., 1994). Sin embargo, el mayor problema que se enfrenta con el control químico de este ácaro es su rápida habilidad para desarrollar resistencia después de unas pocas generaciones (Stumpf et al., 2001; Stumpf y Nauen, 2002). De hecho, el desarrollo de resistencia a acaricidas por parte de T. urticae está ampliamente demostrado a nivel mundial, donde los casos reportados superan los 200, incluyendo acaricidas recientemente autorizados para su control como abamectina (Rizzieri et al., 1988; Georghiou y Lagunes, 1991; Konanz y Nauen, 2004).

En lo que respecta a Chile, se ha reportado resistencia a azinfos metil y metil paration (Georghiou y Lagunes, 1991). Sin embargo, ninguna publicación, nacional o internacional, provienen de T. urticae asociada a ornamentales, por lo que el objetivo de la presente investigación fue evaluar la susceptibilidad a diferentes acaricidas de Tetranychus urticae colectadas en Primula obconica Hance y Convolvulus arvensis $\mathrm{L}$.

\section{MATERIALES Y MÉTODOS}

\section{Recolección de T. urticae Koch}

Los ácaros se colectaron en correhuela (Convolvulus arvensis L., Convolvulaceae) y prímula (Pri- mula obconica Hance, Primulaceae) en la Estación Experimental "El Nogal" de la Facultad de Agronomía de la Universidad de Concepción (36³4' lat. Sur, $76^{\circ} 6^{\prime}$ long. Oeste), en Chillán, Octava Región, entre octubre de 2005 y marzo de 2006. Las plantas de prímula infestadas pertenecían al invernadero de parques y jardines del campus universitario donde las plantas son tratadas con dicofol cada 10 días. Los ácaros colectados en correhuela provinieron de plantas infestadas ubicadas en un patio de almacenamiento de maquinaria agrícola, alejado de las zonas de cultivo, por lo que se asumió que era sensible a cualquier acaricida y podía ser considerada como población de referencia. Los ácaros se reprodujeron en invernadero en plantas de frejol (Phaseolus vulgaris L.) cv. Tórtola sembradas en macetas.

\section{Metodología}

Se utilizó la metodología de Herron et al. (2004), la cual consistió en infestar un disco foliar de frejol de $12 \mathrm{~mm}$ de diámetro con 25 adultos de T. urticae para luego asperjar los acaricidas con una torre de Potter (Potter, 1952). Se evaluaron los acaricidas abamectina (Fast 1,8 EC), azadirachtina (Oikoneem EC), azufre (Azufre $80 \mathrm{WP}$ ), dicofol (Dicofol 25 WP), fenazaquin (Magister 20 SC) y pyridaben (Sanmite WP) que constituyeron siete tratamientos, ya que abamectina se evaluó sola y en mezcla con el surfactante fosfatidilcolina de acuerdo a la recomendación de los fabricantes. Los compuestos activos y modos de acción de los acaricidas evaluados se indican en el Cuadro 1. Las dosis evaluadas fueron $\mathrm{X} / 4, \mathrm{X} / 2, \mathrm{X}, 2 \mathrm{X}$ y $4 \mathrm{X}$, siendo $\mathrm{X}$ la dosis mínima recomendada por el fabricante. Además, se utilizó un testigo absoluto que consistió en agua destilada.

\section{Evaluación de mortalidad}

Se cuantificó la mortalidad de T. urticae a las 6, $12,24,36,48,60,72$ y 84 h después de la aplicación, considerando como muerto al ácaro que no mostraba actividad motora al ser tocado con una aguja de disección (Young-Joon et al., 2004). El porcentaje de mortalidad se corrigió por la fórmula de Abbott (1925).

\section{Análisis Probit}

Se realizó un análisis Probit (Finney, 1971) para obtener las concentraciones letales $50 \%\left(\mathrm{CL}_{50}\right)$ y $90 \%\left(\mathrm{CL}_{90}\right)$ y los tiempos letales $50 \%\left(\mathrm{TL}_{50}\right)$ y $90 \%$ $\left(\mathrm{TL}_{90}\right)$, usando el software Raymond Probit Analysis ${ }^{\circledR}$ (Raymond, 1985). 
Cuadro 1. Acaricidas evaluados en el control de Tetranychus urticae Koch en laboratorio.

Table 1. Acaricides evaluated in Tetranychus urticae Koch control under laboratory conditions.

\begin{tabular}{|c|c|c|c|c|}
\hline $\begin{array}{l}\text { Ingrediente } \\
\text { activo }\end{array}$ & $\begin{array}{l}\text { Nombre } \\
\text { comercial }\end{array}$ & $\begin{array}{c}\text { Grupo } \\
\text { químico }\end{array}$ & Mecanismo de acción & $\begin{array}{c}\text { Dosis } \\
\text { (100 L agua) } \\
\end{array}$ \\
\hline Abamectina & Fast $1,8 \mathrm{EC}$ & Avermectinas & Agonista del ácido gama amino butírico & $80 \mathrm{~mL}$ \\
\hline Azadirachtina & Oikoneem CE-90 & Limonoides & Precursor de la hormona de muda & $1.000 \mathrm{~mL}$ \\
\hline Azufre & Azufre 25 WP & Inorgánicos & Desconocido & $1.000 \mathrm{~g}$ \\
\hline Dicofol & Dicofol 25 WP & Organoclorados & $\begin{array}{l}\text { Cambio de permeabilidad en la } \\
\text { membrana nerviosa }\end{array}$ & $1.000 \mathrm{~g}$ \\
\hline Fenazaquin & Magister $20 \mathrm{SC}$ & Quinazolinas & Inhibidor del transporte de electrones & $45 \mathrm{~mL}$ \\
\hline Pyridaben & Sanmites WP & Piridazinonas & Inhibidor del transporte de electrones & $75 \mathrm{~g}$ \\
\hline
\end{tabular}

\section{Eficacia}

De acuerdo a lo recomendado por Lacey y Singer (1982), se calculó la eficacia, la cual es la relación $\mathrm{CL}_{90} / \mathrm{CL}_{50}$ que indica una mayor eficacia del compuesto evaluado mientras menor sea el cociente.

\section{Índice de resistencia}

Se calculó el índice de resistencia (IR), el cual consiste en la relación entre la $\mathrm{CL}_{50}$ de la población evaluada ( $P$. obconica) y la población de referencia (C. arvensis) (Young-Joon et al. 2004).

\section{Diseño experimental}

El diseño experimental fue completamente al azar. Los datos obtenidos se sometieron a un análisis de varianza y a un test de comparación de medias de Tukey con un nivel de significancia del $95 \%$.

\section{RESULTADOS Y DISCUSIÓN}

\section{Mortalidad \\ Convolvulus arvensis $\mathrm{L}$.}

Los resultados obtenidos muestran que todos los compuestos utilizados en mayor o menor tiempo tuvieron efecto tóxico sobre T. urticae (Cuadro 2). Se destaca la mortalidad obtenida a las $6 \mathrm{~h}$ por abamectina en mezcla con fosfatidilcolina, que superó el $90 \%$, aunque no difiere significativamente ( $\mathrm{p}>$ $0,05)$ de abamectina sola y fenazaquin. Por otra parte, azadirachtina y azufre fueron los tratamientos con un efecto tóxico más lento, alcanzando valores de 76,2 y $99 \%$, respectivamente, a las $84 \mathrm{~h}$ desde la aplicación. La mortalidad de T. urticae provocada por azadirachtina es similar a la obtenida por Knapp y Kashenge (2003) y Martínez et al. (2004), pero difieren de lo señalado por Castagnoli et al. (2005), quienes obtuvieron un $92,5 \%$ de mortalidad a las $72 \mathrm{~h}$.

\section{Primula obconica Hance}

En los ácaros colectados en esta especie, abamectina más fosfatidilcolina nuevamente logró el mayor porcentaje de mortalidad con prácticamente un $100 \%$ a las $12 \mathrm{~h}$, siendo estadísticamente igual con abamectina sola y diferente a los restantes acaricidas. Los menores niveles de mortalidad, al igual que en el caso de la población colectada en correhuela, fueron de azadirachtina, azufre y pyridaben con valores de $74 ; 87,2$ y 97,6\% respectivamente a las $84 \mathrm{~h}$. Cabe destacar que dicofol produjo una mortalidad de $78,5 \%$ a las $24 \mathrm{~h}$, valor que clasifica a la población como sensible, ya que Saito et al. (1983) señalan que una población es resistente cuando la mortalidad es inferior a $43 \%$ a las $24 \mathrm{~h}$ de la aplicación.

\section{Concentración letal $50 \%\left(\mathrm{CL}_{50}\right)$ y $90 \%\left(\mathrm{CL}_{90}\right)$}

De acuerdo a los resultados obtenidos en el análisis Probit, fenazaquin es el tratamiento que tiene una menor $\mathrm{CL}_{50}$, con $0,11 \mathrm{~mL} \mathrm{~L}^{-1}$ en ambas poblaciones, lo cual demuestra la alta toxicidad de este compuesto para T. urticae (Cuadro 3). Respecto a abamectina sola y en mezcla con fosfatidilcolina, prácticamente no hubo diferencias en sus $\mathrm{CL}_{50}$ en ambas poblaciones, indicando que la acción lograda por el surfactante fue casi nula. También se destaca la $\mathrm{CL}_{50}$ obtenida por dicofol para la población colectada en $P$. obconica que fue de $1,57 \mathrm{~g} \mathrm{~L}^{-1}$ valor muy cercano al $1,83 \mathrm{~g} \mathrm{~L}^{-1}$ que según Saito et al. (1983) tuvo una población con resistencia incipiente. Los valores más elevados de $\mathrm{CL}_{50}$ y $\mathrm{CL}_{90}$ se obtuvieron con azufre y azadirachtina, lo cual indica que a pesar de que ambos tienen algún grado de toxicidad para T. urticae, éste es menor a los otros tratamientos evaluados. La alta $\mathrm{CL}_{50}$ del azufre podría explicarse con lo señalado por Deirdre et al. (2005), quienes indican que este compuesto tiene 


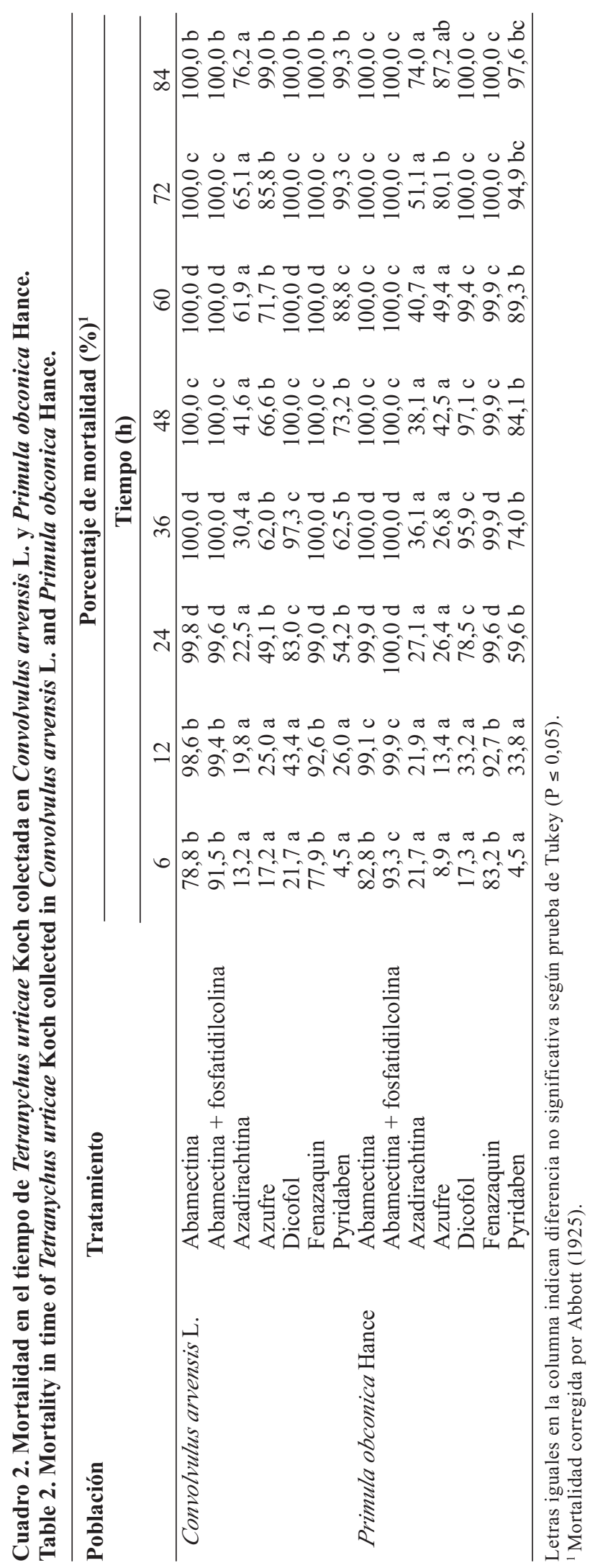

una alta toxicidad para ácaros pero una lenta acción, por lo que es más relevante el tiempo letal que la concentración.

\section{Eficacia}

La mayor eficacia se obtuvo con abamectina en mezcla con fosfatidilcolina con valores de 1,6 y 1,9 para la población colectada en $C$. arvensis y P. obconica, respectivamente (Cuadro 3). Esto contrasta con azadirachtina y azufre, los cuales mostraron la menor eficacia tanto en $C$. arvensis como $P$. obconica. Es decir, estos dos tratamientos requieren de un mayor aumento de su concentración para alcanzar un $90 \%$ de control en relación a los otros tratamientos. Este antecedente cobra especial importancia en azadirachtina, pues los agroquímicos formulados con este compuesto presentes en el mercado nacional tienen un costo más alto que el azufre, lo cual puede convertirlos en una alternativa de baja rentabilidad.

\section{Tiempo letal $50 \%\left(\mathrm{TL}_{50}\right)$ y tiempo letal $90 \%$ $\left(\mathrm{TL}_{90}\right)$}

Los menores tiempos letales $50 \%\left(\mathrm{TL}_{50}\right)$ y $90 \%$ $\left(\mathrm{TL}_{90}\right)$ de ambas poblaciones fueron obtenidos por abamectina sola y en mezcla con fosfatidilcolina (Cuadro 3), lo cual confirma los resultados obtenidos en la medición de eficacia, ya que estos tratamientos eliminaron a T. urticae en un corto plazo en todos los bioensayos. Por el contrario, azadirachtina y azufre necesitarían más de 64 y $82 \mathrm{~h}$ para matar al 50 y $90 \%$ de la población, respectivamente. Sin embargo, en la relación $\mathrm{TL}_{50} / \mathrm{TL}_{90}$ en ambas poblaciones estos dos tratamientos presentaron el menor valor, lo que sugiere que a pesar de necesitar mayor tiempo para eliminar a la población, azadirachtina y azufre requerirían un menor tiempo para pasar de 50 a $90 \%$ de control, corroborando que el efecto tóxico de estos compuestos se va incrementando en el tiempo.

\section{Índice de resistencia}

Los resultados para el índice de resistencia indican que no existe un elevado nivel de resistencia de T. urticae a ninguno de los acaricidas evaluados (Cuadro 4), y aunque llama la atención el valor alcanzado por azufre, según la escala indicada por Young-Joon et al. (2004) la población colectada en $P$. obconica debe ser considerada como susceptible. Sin embargo, por ser este índice el que presenta un mayor valor, en el manejo de este ácaro, por prevención de un futuro caso de resis- 
Cuadro 3. Concentración letal $50 \%\left(\mathrm{CL}_{50}\right)$ y $90 \%\left(\mathrm{CL}_{90}\right)$, tiempo letal $50 \%\left(\mathrm{TL}_{50}\right)$ y $90 \%\left(\mathrm{TL}_{90}\right)$ y eficacia para dos poblaciones de Tetranychus urticae Koch en laboratorio.

Table 3. Lethal concentration $50 \%\left(\mathrm{LC}_{50}\right)$ and $90 \%\left(\mathrm{LC}_{90}\right)$, lethal time $50 \%\left(\mathrm{LT}_{50}\right)$ and $\mathbf{9 0} \%\left(\mathrm{LT}_{90}\right)$ and efficacy for two poblations of Tetranychus urticae Koch under laboratory conditions.

\begin{tabular}{|c|c|c|c|c|c|c|c|}
\hline \multirow{2}{*}{$\begin{array}{l}\text { Concentración } \\
\text { letal }^{1} \\
\text { (CL) }\end{array}$} & \multirow[b]{2}{*}{ Tratamiento } & \multicolumn{3}{|c|}{ Convolvulus arvensis $\mathrm{L}$. } & \multicolumn{3}{|c|}{ Primula obconica Hance } \\
\hline & & $\mathbf{C} \mathbf{L}_{50}$ & $\mathbf{C L}_{90}$ & Eficacia & $\mathbf{C L} \mathbf{L}_{50}$ & $C L_{90}$ & Eficacia \\
\hline & Abamectina & 1,28 & 4,70 & 3,7 & 1,42 & 2,93 & 2,1 \\
\hline & Abamectina + fosfatidilcolina & 2,55 & 4,92 & 1,9 & 2,54 & 4,10 & 1,6 \\
\hline & Azadirachtina & $15.793,10$ & $2.457 .100,0$ & 155,6 & $1.785,3$ & $131.592,0$ & 73,7 \\
\hline & Azufre & $1.393,50$ & $9.621 .562,0$ & $6.904,4$ & $4.607,8$ & $707.706,0$ & 153,59 \\
\hline & Dicofol & 0,86 & 4,94 & 5,7 & 1,57 & 7,21 & 4,6 \\
\hline & Fenazaquin & 0,11 & 0,29 & 2,7 & 0,11 & 0,29 & 2,7 \\
\hline & Pyridaben & 1,14 & 3,46 & 3,0 & 1,30 & 5,91 & 4,56 \\
\hline \multirow[t]{8}{*}{$\begin{array}{l}\text { Tiempo letal (h) } \\
\text { (TL) }\end{array}$} & Tratamiento & $\mathbf{T L}_{50}$ & $\mathrm{TL}_{90}$ & Eficacia & $\mathrm{TL}_{50}$ & $\mathrm{TL}_{90}$ & Eficacia \\
\hline & Abamectina & 5,0 & 12,1 & 2,4 & 4,5 & 9,2 & 2,0 \\
\hline & Abamectina + fosfatidilcolina & 3,1 & 9,7 & 3,1 & 3,1 & 6,7 & 2,2 \\
\hline & Azadirachtina & 79,5 & 120,3 & 1,5 & 80,8 & 105,4 & 1,3 \\
\hline & Azufre & 68,3 & 82,3 & 1,2 & 64,5 & 87,9 & 1,4 \\
\hline & Dicofol & 22,8 & 42,6 & 1,9 & 24,0 & 42,8 & 1,8 \\
\hline & Fenazaquin & 6,1 & 15,6 & 2,6 & 4,1 & 13,7 & 3,3 \\
\hline & Pyridaben & 51,4 & 66,9 & 1,3 & 53,2 & 77,8 & 1,5 \\
\hline
\end{tabular}

${ }^{1}$ Valores en $\mathrm{g} \mathrm{L}^{-1} \mathrm{o} \mathrm{mL} \mathrm{L}^{-1}$ de producto comercial.

Cuadro 4. Índice de resistencia (IR) para una población de Tetranychus urticae Koch colectada en Primula obconica Hance.

Table 4. Resistance index (IR) for a Tetranychus urticae Koch population collected in Primula obconica Hance.

\begin{tabular}{lc}
\hline Tratamiento & IR \\
\hline Abamectina & 1,11 \\
Abamectina + fosfatidilcolina & 1,11 \\
Azadirachtina & 0,11 \\
Azufre & 3,31 \\
Dicofol & 1,83 \\
Fenazaquin & 1,00 \\
Pyridaben & 1,14 \\
\hline
\end{tabular}

tencia se debiera preferir alguno de los otros acaricidas evaluados.

\section{CONCLUSIONES}

La población de Tetranychus urticae Koch colectada en Primula obconica es sensible a abamectina, azadirachtina, azufre, dicofol, fenazaquin y pyridaben.

Abamectina sola y en mezcla con fosfatidilcolina y fenazaquin, tienen un alto efecto derribante sobre Tetranychus urticae Koch. 


\section{LITERATURA CITADA}

Abbott, W.S. 1925. A method of computing the effectiveness of an insecticide. J. Econ. Entomol. 18:265-267.

Castagnoli, M., M. Liguori, S. Simoni, and C. Duso. 2005. Toxicity of some insecticides to Tetranychus urticae, Neoseiulus californicus and Tydeus californicus. BioControl 50:611-622.

Deirdre, A.P., D.G. James, C.L. Wright, D.T. Roak, and W.E. Snyder. 2005. Effects of chlorpyrifos and sulfur on spider mites (Acari: Tetranychidae) and their natural enemies. Biol. Control 33:324-334.

Finney, D. 1971. Probit analysis. 333 p. Cambridge University Press, Cambridge, UK.

Georghiou, G., and A. Lagunes. 1991. The occurrence of resistance to pesticides in arthropods. 318 p. FAO/ University of California, Riverside, California, USA.

González, R.H. 1989. Insectos y ácaros de importancia agrícola y cuarentenaria en Chile. 310 p. Universidad de Chile, Santiago, Chile.

Herron, G.A., J. Rophail, and L.J. Wilson. 2004. Chlorfenapyr resistance in two-spotted spider mite (Acari: Tetranychidae) from Australian cotton. Exp. Appl. Acarol. 34:315-321.

Knapp, M., and S.S. Kashenge. 2003. Effects of different neem formulations on the twospotted spider mite, Tetranychus urticae Koch, on tomato (Lycopersicon esculentum Mill.). Insect Sci. Appl. 23:1-7.

Konanz, S., and R. Nauen. 2004. Purification and partial characterization of a glutathione S-transferase from the two-spotted spider mite, Tetranychus urticae. Pestic. Biochem. Physiol. 79:49-57.

Lacey, L., and S. Singer. 1982. Larvicidal activity of new isolates of Bacillus sphaericus and Bacillus thuringiensis $(\mathrm{H}-14)$ against anopheline and culicine mosquitoes. Mosquito News 42:537-543.

Martínez, E., F. Sáenz, F. Moreno, V. Marco, and I. Pérez. 2004. Effects of azadirachtin on the two-spotted spider mite, Tetranychus urticae (Acari: Tetranychidae). Exp. Appl. Acarol. 35:215-222.

Potter, C. 1952. An improved laboratory apparatus for applying direct sprays and surface films, with data on electrostatic charge on atomized spray fluids. Ann. Appl. Biol. 39:1-29.
Raymond, M. 1985. Présentation d'un programme d'analyse log-probit pour micro-ordinateur. Entomol. Med. Parasitol. 22:117-121.

Rizzieri, D.A., T.J. Dennehy, and T.J. Glover. 1988. Genetic analysis of Dicofol resistance in two populations of Twospotted spider mite (Acari: Tetranychidae) from New York apple orchards. J. Econ. Entomol. 81:1271-1276.

Sadrás, V.O., L.J. Wilson, and D.A. Rally. 1998. Water deficit enhanced cotton resistance to spider mite herbivory. Ann. Bot. (London) 81:273-286.

Saito, T., K. Tabata, and S. Kohno. 1983. Mechanisms of acaricide resistance with emphasis on Dicofol. p. 429444. In Georghiou, G.P., and T. Saito. (eds.). Pest resistance to pesticides. Plenum Press, New York, USA.

Stumpf, N., P.W. Claus, W. Kraus, G.D. Moores, and R. Nauen. 2001. Resistance to organophosphates and biochemical genotyping of acetylcholinesterases in Tetranychus urticae (Acari: Tetranychidae). Pestic. Biochem. Physiol. 69:131-142.

Stumpf, N., and R. Nauen. 2002. Biochemical markers linked to abamectin resistance in Tetranychus urticae (Acari: Tetranychidae). Pestic. Biochem. Physiol. 72:111-121.

Takematsu, A.P., N.S. Filho, M.F. de Souza Filho, y M.E. Sato. 1994. Sensibilidade de Tetranychus urticae (Koch, 1836) proveniente de roseira (Rosa sp.) de Holambra-SP a alguns acaricidas. Rev. Agric. (Piracicaba) 69(2):129-137.

University of California. 2002. Integrated pest management for floriculture and nurseries. Publication 3402. 422 p. University of California, Division of Agriculture and Natural Resources, Oakland, California, USA.

Young-Joon, K., L. Si-Hyeock, L. Si-Woo and A. YoungJoon. 2004. Fenpyroximate resistance in Tetranychus urticae (Acari:Tetranychidae): cross-resistance and biochemical resistance mechanisms. Pest. Manag. Sci. 60:1001-1006. 